\title{
Identification of microRNA profiles associated with refractory primary biliary cirrhosis
}

\author{
TEPPEI SAKAMOTO ${ }^{1}$, ASAHIRO MORISHITA $^{1}$, TAKAKO NOMURA ${ }^{1}$, JOJI TANI $^{1}$, HISAAKI MIYOSHI ${ }^{1}$, \\ HIROHIRO YONEYAMA ${ }^{1}$, HISAKAZU IWAMA ${ }^{2}$, TAKASHI HIMOTO ${ }^{3}$ and TSUTOMU MASAKI $^{1}$ \\ ${ }^{1}$ Department of Gastroenterology and Neurology; ${ }^{2}$ Life Science Research Center, Kagawa University Faculty of Medicine, \\ Miki-cho, Kagawa 761-0793; ${ }^{3}$ Department of Medical Technology, Kagawa Prefectural University of Health Sciences, \\ Takamatsu, Kagawa 761-0123, Japan
}

Received June 8, 2015; Accepted June 23, 2016

DOI: $10.3892 / \mathrm{mmr} .2016 .5606$

\begin{abstract}
MicroRNAs (miRNAs) are small, endogenous, non-coding RNAs that control the target gene translation by RNA interference; miRNAs are associated with cellular processes, including proliferation, differentiation, apoptosis, and cell survival. Primary biliary cirrhosis (PBC) is a chronic cholestatic liver disease of unknown etiology. One third of patients with $\mathrm{PBC}$ demonstrate suboptimal responses, which result in worse outcomes. It has been previously reported that miRNAs are involved in drug resistance, however, the association between miRNA expression levels and refractory PBC remains to be fully elucidated. In the present study, among the 20 patients with PBC treated with ursodeoxycholic acid or bezafibrate, 15 patients were classed as treatment-effective, and 5 were classed as being treatment-resistant. Using the miRNA array technique, miRNA profiles were identified for each group. A total of 35 miRNAs were significantly upregulated, and 23 were significantly downregulated in the treatment-resistant group compared with the treatment-effective group. In order to examine the association between the highly altered miRNAs and clinical features of the two groups, numerous parameters were analyzed. Elevated levels of direct bilirubin, aspartate transaminase (AST), and alanine transaminase (ALT) were identified to be associated with miRNA-122 upregulation. AST, ALT, and $\gamma$ guanosine triphosphate were additionally associated with miRNA-378f upregulation. However, the reduction of miRNA-4311 was associated with reduced levels of AST and ALT. miRNA-4714-3p was also negatively
\end{abstract}

Correspondence to: Professor Tsutomu Masaki, Department of Gastroenterology and Neurology, Kagawa University Faculty of Medicine, 1750-1 Ikenobe, Miki-cho, Kita-gun, Kagawa 761-0793, Japan

E-mail: tmasaki@med.kagawa-u.ac.jp

\section{Abbreviations: UDCA, ursodeoxycholic acid}

Key words: primary biliary cirrhosis, microRNA, treatment resistancy, ursodeoxycholic acid, bezafibrate correlated with total bilirubin and lactate dehydrogenase. Therefore, identifying the miRNA profile was demonstrated to be a useful approach in the characterization of PBC development. It is suggested that highly altered miRNAs may be potential biomarkers for use in the development of treatment of patients with refractory PBC.

\section{Introduction}

MicroRNAs (miRNAs) are small, non-coding RNAs that are 21-30 nucleotides in length and that interfere with their target mRNAs, and there are approximately 2,000 of these sequences in the human genome (1). Each miRNA negatively regulates protein translation through the degradation of mRNA cleaved by a miRNA-associated RNA-induced silencing complex (2). It is now apparent that an individual miRNA can modulate more than 200 miRNAs $(3,4)$, and greater than 2,000 miRNAs have been identified at present (5).

Previous studies have demonstrated that miRNAs can serve an important role in autoimmune diseases (AID) (6), including systemic lupus erythematosus (7) and rheumatoid arthritis (8). Several miRNAs have been associated with the maturation of various immune cells and with the regulation of their functions (9-11), suggesting that certain miRNAs may affect the development and etiology of AID. In addition, although it has been demonstrated that miRNAs are involved in numerous types of liver disease, the association between miRNA profiles and autoimmune liver diseases, including the clinical relevance of miRNA in treating these diseases, remains unclear.

Primary biliary cirrhosis (PBC) is a slow, progressive, chronic cholestatic disease that is characterized by the destruction of the intrahepatic bile ducts and fibrosis, and with portal inflammation, it may develop into liver cirrhosis (12). Several processes are observed during the development of PBC: i) A specific immune response including antimitochondrial antibodies (AMA), which are directed towards the E2 component of the 2-oxo-dehydrogenase pathway, particularly towards the pyruvate dehydrogenase-E2 complex; ii) abnormal innate immunity, in which the ligands of the Toll-like receptors 3 and 4 stimulate type $1 \mathrm{~T}$ helper cell responses, resulting in biliary cell destruction; and iii) a higher frequency autoreactive T cell 
Table I. Characteristics of the study groups.

\begin{tabular}{lcc}
\hline Characteristic & Treatment-effective group $(\mathrm{n}=15)$ & Treatment-resistant group (n=5) \\
\hline Gender (F/M) & $15 / 0$ & $4 / 1$ \\
Age (years) & $59.3 \pm 9.0$ & $40 \pm 14.6$ \\
TP (mg/dl) & $7.79 \pm 0.62$ & $7.82 \pm 0.37$ \\
Alb (mg/dl) & $4.01 \pm 0.35$ & $3.9 \pm 0.44$ \\
T-bil (mg/dl) & $0.61 \pm 0.24$ & $0.88 \pm 0.48$ \\
D-bil (mg/dl) & $0.21 \pm 0.15$ & $0.32 \pm 0.11$ \\
AST (IU/l) & $75 \pm 66.8$ & $87.6 \pm 50.8$ \\
ALT (IU/l) & $94.9 \pm 131.7$ & $101.6 \pm 52.7$ \\
ALP (IU/l) & $602.8 \pm 282.2$ & $1080.4 \pm 504.4$ \\
LDH (IU/l) & $246.1 \pm 56.5$ & $246.1 \pm 65.7$ \\
$\gamma$-GTP (IU/l) & $215.5 \pm 140.3$ & $399 \pm 277.1$ \\
IgA (mg/dl) & $272.4 \pm 124.6$ & $341.2 \pm 140.5$ \\
IgG (mg/dl) & $1908.3 \pm 598.2$ & $1955.4 \pm 810.8$ \\
IgM (mg/dl) & $381.9 \pm 258.4$ & $404.4 \pm 126.1$ \\
AMA (positive/negative) & $9 / 6$ & $3 / 2$ \\
AMA-M2 (positive/negative) & $11 / 4$ & $3 / 2$
\end{tabular}

F, female; M, male; TP, total protein; Alb, albumin; T-bil, total bilirubin; D-bil, direct bilirubin; AST, aspartate transaminase; ALT, alanine transaminase; ALP, alkaline phosphatase; LDH, lactate dehydrogenase; $\gamma$-GTP, $\gamma$-guanosine triphosphate; Ig, immunoglobulin; AMA, antimitochondrial antibody; AMA-M2, AMA M2 subtype.

precursor in the liver $(13,14)$. During PBC treatment, it has been demonstrated that patients with efficient biochemical responses to ursodeoxycholic acid (UDCA) have a low risk of developing liver failure or cirrhosis in the long term (15). In addition, the normalization of alanine transaminase (ALT) with additional bezafibrate treatment reduces the risk of occurrence of liver-associated symptoms in patients with PBC with insufficient responses to UDCA (16). At present, no biomarker has been discovered for the prediction of refractory PBC. Notably, alterations in hepatic miRNAs, including miRNA-122a, miRNA-26a, miRNA-328 and miRNA-299-5p, have been previously associated with PBC (17). However, the association between these alterations and refractory $\mathrm{PBC}$ remains to be fully elucidated.

The aim of the current study was to identify the miRNA profiles associated with drug resistance to UDCA, bezafibrate and prednisolone, in addition to the various clinical parameters in patients with $\mathrm{PBC}$.

\section{Materials and methods}

Patients. The current study involved 20 patients with PBC treated at Kagawa University Hospital (Miki-Cho, Japan) between 2001 and 2013. The PBC diagnosis was established when two of the following three criteria were met: i) Biochemical evidence of cholestasis, primarily based on elevated alkaline phosphatase levels (ALP), ii) the presence of AMA, and iii) histological evidence of nonsuppurative destructive cholangitis and destruction of the interlobular bile ducts (12).

Clinical presentation. The treatment-effective group was defined by reductions in ALP (less than $600 \mathrm{IU} / 1$ ) and $\gamma$-guanosine triphosphate $(\gamma$-GTP) (less than $100 \mathrm{IU} / \mathrm{l})$ within a year of being treated with UDCA at a maximum dose of $900 \mathrm{mg} /$ day. Bezafibrate administration was decided upon within a year subsequent to initiation of the UDCA medication, in accordance with the response to UDCA monotherapy $(n=8)$. The treatment-resistant group included the patients who did not meet a condition of the treatment-effective group. Subsequent to undergoing continuous treatment more than a year, serum samples were collected from those patients. The baseline characteristics of $\mathrm{PBC}$ at the time of miRNA sampling are presented in Table I. The present study was approved by the ethics committee of Kagawa University Faculty of Medicine, and informed consent was obtained from all patients.

Analysis of the microRNA array. Total RNA was extracted from the samples derived from the serum samples using a miRNeasy Mini kit (Qiagen GmbH, Hilden, Germany), according to the manufacturer's instructions. RNA samples typically exhibited $A_{260 / 280}$ ratios between 1.9 and 2.1, which were measured using an Agilent 2100 Bioanalyzer (Agilent Technologies, Inc., Santa Clara, CA, USA).

Subsequent to measuring the RNA with an RNA 6000 Nano kit (Agilent Technologies, Inc.), the samples were labeled using a miRCURY Hy3/Hy5 Power Labeling kit (Takara Bio, Inc., Otsu, Japan) and were hybridized onto a human miRNA Oligo chip (version 19.0; Toray Industries, Inc., Tokyo, Japan). Scanning was performed with the 3D-Gene Scanner 3000 (Toray Industries, Inc.), and 3D-Gene Extraction software (version 1.2; Toray Industries, Inc.) was used to read the raw intensity of the image. To determine the alterations in miRNA expression between the treatment-resistant and treatment-effective groups, the raw data were analyzed with 
Table II. Statistical results of the miRNAs that were upregulated in the serum of patients with primary biliary cirrhosis.

Fold

Upregulated miRNA

P-value

(resistant/effective group)

hsa-let-7b

0.026804

1.567847

hsa-let-7f- $1^{*}$

0.047298

1.215035

hsa-miR-122

0.026881

1.561976

hsa-miR-1233

0.038244

1.208806

hsa-miR-1260

0.041029

1.227792

hsa-miR-136*

0.037889

1.249572

hsa-miR-150

0.039072

1.253923

hsa-miR-2053

0.039291

1.182613

hsa-miR-210

0.003102

1.564477

hsa-miR-218-1*

0.045877

1.240002

hsa-miR-2467-3p

0.035871

1.246314

hsa-miR-3065-3p

0.003962

1.417027

hsa-miR-3123

0.043136

1.298624

hsa-miR-3173-5p

0.025762

1.276267

hsa-miR-3616-3p

0.028351

1.273818

hsa-miR-378f ${ }^{\mathrm{a}}$

0.013278

1.527677

hsa-miR-378g

0.022622

1.330441

hsa-miR-3976

0.014323

1.320455

hsa-miR-409-5p

0.030094

1.220102

hsa-miR-4424

0.041228

1.236549

hsa-miR-4535

0.007167

1.394004

hsa-miR-4655-3p

0.005221

1.25409

hsa-miR-4670-3p

0.007172

1.489381

hsa-miR-4781-3p

1.290433

hsa-miR-504

0.012059

1.349747

hsa-miR-509-3p

0.026989

1.333371

hsa-miR-511

0.037295

1.348944

hsa-miR-542-5p

0.01903

1.344654

hsa-miR-548ac

0.03619

1.230733

hsa-miR-548f

0.035305

1.239292

hsa-miR-610

0.049632

1.228792

hsa-miR-612

0.026332

1.251725

hsa-miR-650

0.048399

1.182034

hsa-miR- $659^{a}$

0.02361

1.500267

hsa-miR-802

0.001558

1.305961

${ }^{a}$ Five miRNAs were significantly upregulated greater than 1.5 times in the treatment-resistant group as compared with the treatment-effective group. miRNA/miR, microRNA.

GeneSpring GX software, version 10.0 (Agilent Technologies, Inc.). Samples were first normalized relative to the 28S RNA, and then the baseline was corrected to the median of all samples.

Replicate data were consolidated into two groups: The treatment-resistant and treatment-effective groups. Hierarchical clustering was performed with the farthest neighbor method using the absolute Pearson's correlation coefficient as a metric. The base-2 log-transformed intensities were median-centered for each row (miRNA probe) and were color-coded, as presented on the heat map. The P-value cutoff was set to 0.05 . Only alterations greater than
$50 \%$ in a minimum of one of the time points for each sample were considered to be significant. All analyzed data were scaled by global normalization. The statistical significance of the differentially expressed miRNAs was analyzed with Student's t-test.

Statistical analysis. Statistical analyses were performed using the computer-assisted StatFlex software, version 6.0 (Artec Co., Ltd., Osaka, Japan). A paired analysis between the groups was conducted using Student's t-test and Pearson's correlation coefficient. $\mathrm{P}<0.05$ was considered to indicate a statistically significant difference. 
Table III. Statistical results of the miRNAs that were downregulated in the serum of the patients with primary biliary cirrhosis.

\begin{tabular}{|c|c|c|}
\hline Downregulated miRNA & P-value & $\begin{array}{c}\text { Fold } \\
\text { (resistant/effective group) }\end{array}$ \\
\hline hsa-miR-125b-2* & 0.014229 & 0.7001 \\
\hline hsa-miR-1301 & 0.031384 & 0.77713 \\
\hline hsa-miR-2277-3p & 0.02819 & 0.772271 \\
\hline hsa-miR-3136-3p $\mathrm{p}^{\mathrm{a}}$ & 0.003916 & 0.662795 \\
\hline hsa-miR-33b* & 0.013788 & 0.815009 \\
\hline hsa-miR-340* & 0.008022 & 0.78203 \\
\hline hsa-miR-3675-5p & 0.015949 & 0.685806 \\
\hline hsa-miR-3689a-3pa & 0.015287 & 0.659541 \\
\hline hsa-miR-3690a & 0.017489 & 0.614312 \\
\hline hsa-miR-380* & 0.018128 & 0.755665 \\
\hline hsa-miR-4260 & 0.046847 & 0.753042 \\
\hline hsa-miR-4311 a & 0.044072 & 0.659274 \\
\hline hsa-miR-4423-5p & 0.035972 & 0.773386 \\
\hline hsa-miR-4504 & 0.030226 & 0.696011 \\
\hline hsa-miR-4655-5p & 0.038655 & 0.752697 \\
\hline hsa-miR-4714-3p $\mathrm{p}^{\mathrm{a}}$ & 0.007293 & 0.655707 \\
\hline hsa-miR-4720-3p & 0.027112 & 0.719039 \\
\hline hsa-miR-520a-5p & 0.012847 & 0.732509 \\
\hline hsa-miR-548z & 0.046249 & 0.746099 \\
\hline hsa-miR-602 & 0.003259 & 0.722861 \\
\hline hsa-miR-607 & 0.043153 & 0.796838 \\
\hline hsa-miR-770-5p & 0.012182 & 0.719448 \\
\hline hsa-miR-873 & 0.040941 & 0.769026 \\
\hline
\end{tabular}

${ }^{\mathrm{a}} \mathrm{A}$ total of 5 miRNAs were significantly downregulated less than 0.67 times in the treatment-resistant group compared with the treatment-effective group. miRNA/miR, microRNA.

Table IV. Association between representative upregulated miRNAs and clinical parameters in patients with primary biliary cirrhosis.

\begin{tabular}{|c|c|c|c|c|c|}
\hline Parameter & let- $7 b$ & miR-122 & miR-210 & miR-378f & $\operatorname{miR}-659$ \\
\hline $\mathrm{TP}$ & 0.1038 & 0.4242 & 0.095 & 0.281 & 0.0973 \\
\hline Alb & 0.1084 & 0.2757 & -0.0793 & -0.1152 & -0.1483 \\
\hline T-Bil & 0.0636 & 0.1186 & -0.0581 & -0.1196 & -0.1074 \\
\hline D-Bil & 0.3542 & $0.4811^{\mathrm{a}}$ & 0.1879 & 0.0569 & -0.0729 \\
\hline AST & 0.3694 & $0.5539^{a}$ & 0.2774 & $0.4814^{\mathrm{a}}$ & 0.3036 \\
\hline ALT & $0.4883^{\mathrm{a}}$ & $0.7018^{\mathrm{a}}$ & 0.3105 & $0.5642^{\mathrm{a}}$ & 0.3424 \\
\hline ALP & 0.3182 & 0.3793 & 0.2231 & 0.2928 & 0.0445 \\
\hline LDH & -0.3468 & -0.2167 & -0.16 & -0.1538 & -0.3315 \\
\hline$\gamma$-GTP & 0.2733 & 0.5706 & 0.296 & $0.5359^{a}$ & $0.4495^{\mathrm{a}}$ \\
\hline $\mathrm{IgG}$ & -0.1361 & 0.0219 & -0.0543 & 0.2627 & 0.1534 \\
\hline $\operatorname{IgM}$ & 0.0957 & 0.1882 & 0.0436 & -0.0893 & -0.0768 \\
\hline
\end{tabular}

${ }^{a} \mathrm{P}<0.05$. miRNA/miR, microRNA; TP, total protein; Alb, albumin; T-bil, total bilirubin; D-bil, direct bilirubin; AST, aspartate transaminase; ALT, alanine transaminase; ALP, alkaline phosphatase; LDH, lactate dehydrogenase; $\gamma$-GTP, $\gamma$-guanosine triphosphate; Ig, immunoglobulin.

\section{Results}

miRNA expression in the serum of patients with PBC.
miRNAs are present in human serum and are highly stable due to the resistance to RNase digestion (18). An miRNA array was performed in the current study using the serum 
Table V. Association between representative downregulated miRNAs and clinical parameters in patients with primary biliary cirrhosis.

\begin{tabular}{lccccc}
\hline Parameter & miR-3136-3p & miR-3689a-3p & miR-3690 & miR-4311 & -0.1489 \\
\hline TP & -0.1468 & -0.1996 & -0.2365 & -0.3657 & -0.0633 \\
Alb & 0.0189 & -0.1204 & -0.2857 & 0.2015 & 0.0557 \\
T-Bil & 0.3531 & 0.0764 & -0.2528 & -0.0678 & $0.4763^{\mathrm{a}}$ \\
D-Bil & 0.1435 & -0.2297 & -0.3576 & $-0.5766^{\mathrm{a}}$ & 0.3744 \\
AST & -0.0728 & -0.3675 & -0.1569 & $-0.559^{\mathrm{a}}$ & 0.01138 \\
ALT & -0.1218 & $-0.458^{\mathrm{a}}$ & -0.2569 & -0.3857 & 0.2868 \\
ALP & 0.1701 & -0.1837 & -0.1857 & -0.1767 & $-0.5841^{\mathrm{a}}$ \\
LDH & 0.3869 & 0.3149 & -0.3301 & -0.4519 & -0.0204 \\
Y-GTP & -0.3012 & -0.3801 & 0.2479 & -0.2368 & -0.3262 \\
IgG & -0.2189 & -0.0718 & -0.2388 & -0.2487 & \\
IgM & -0.0193 & 0.0584 & & & -0.0262 \\
\hline
\end{tabular}

${ }^{\text {aP }}<0.05$. miRNA/miR, microRNA; TP, total protein; Alb, albumin; T-bil, total bilirubin; D-bil, direct bilirubin; AST, aspartate transaminase; ALT, alanine transaminase; ALP, alkaline phosphatase; LDH, lactate dehydrogenase; $\gamma$-GTP, $\gamma$-guanosine triphosphate; Ig, immunoglobulin.

of patients with PBC. The expression patterns of 1,769 miRNAs were examined using extracted serum miRNAs. As presented in Table II, 35 miRNAs were significantly upregulated in the treatment-resistant group compared with the treatment-effective group. However, 23 miRNAs in the treatment-resistant group were significantly downregulated compared with those in the treatment-effective group (Table III). In addition, unsupervised hierarchical clustering analysis, using Pearson's correlation, demonstrated that the treatment-effective group was clustered separately from the treatment-resistant group (Fig. 1). Furthermore, highly altered miRNAs (greater than 1.5-fold) were analyzed, and the two groups were clustered separately using highly altered miRNAs (Fig. 2 and Tables I and II).

miRNA expression and clinical parameters. To examine the association between highly altered miRNAs and clinical features, various parameters were analyzed. As presented in Table IV, elevated levels of direct bilirubin (D-bil), aspartate transaminase (AST), and ALT were associated with miRNA-122 upregulation (Table IV). AST, ALT, and $\gamma$-GTP were also related to miRNA-378f upregulation (Table IV). However, the reduction of miRNA-4311 was related to the decreases of AST and ALT (Table V). miRNA-4714-3p was also negatively correlated to total bilirubin and lactate dehydrogenase (Table V). These results suggest that these miRNAs may be new biomarkers for the development of PBC.

\section{Discussion}

It has been demonstrated that patients with $\mathrm{PBC}$ with efficient biochemical responses to UDCA or bezafibrate have low risks of developing liver failure or cirrhosis in the long term $(15,16)$. However, one third of patients with PBC exhibited suboptimal responses to these treatment strategies (19), and these patients demonstrated poorer outcomes (20-22). At present, no useful biomarker has been reported for the development of PBC. In the present study, miRNA expression patterns in the treatment-resistant group markedly differed compared with those in the treatment-effective group. The expression levels of highly altered miRNAs in the resistant group were also associated with the clinical parameters of PBC. Notably, miRNA-122 was significantly upregulated and was associated with D-bil, AST, and ALT in Tables II and IV. Roderburg et al (23) demonstrated that elevated miRNA-122 serum levels are a potent and independent marker of liver injury and are not seen in patients with liver cirrhosis without ongoing liver damage. In addition, it has also been reported that miRNA-122 is upregulated in acute and chronic liver injury (24), myocardial infarction (25) and cancer (26). These results support those indicating that miRNA-122 was enhanced in the serum of the treatment-resistant group, due to the fact that clinical parameters, including AST and ALT, were raised sufficiently to indicate ongoing inflammation in the liver.

In addition, several miRNAs were identified to be associated with cell cycle arrest, including let-7 and miR-210 among the upregulated miRNAs in the treatment-resistant group. It has been previously demonstrated that let-7 and miR-210 targeted cell cycle regulatory molecules $(27,28)$. Han et al (29) reported that miR-194 suppresses cell proliferation through the inhibition of insulin-like growth factor receptor 1 . These reports suggest that various miRNAs, including let-7, miR-210 and miR-194, may inhibit hepatocyte regeneration by regulating cell cycle and cell proliferation in the treatment control group.

Downregulated miRNAs, including miR-125b, miR-380 and miR-602, were detected in the treatment-resistant group. Notably, it has been previously reported that miR-125b and miR-380 are negative regulators of p53 $(30,31)$ and miR-602 regulates the tumor-suppressive gene Ras-association domain family 1 , isoform A (32). This suggests that downregulation of miR-125b, miR-380 and miR-602 may suppress cell proliferation and cell cycle acceleration, and induce apoptosis in the hepatocytes of treatment-resistant patients.

It has been previously reported that miRNAs are able to regulate the efficacy of drugs interacting with the miRNA 


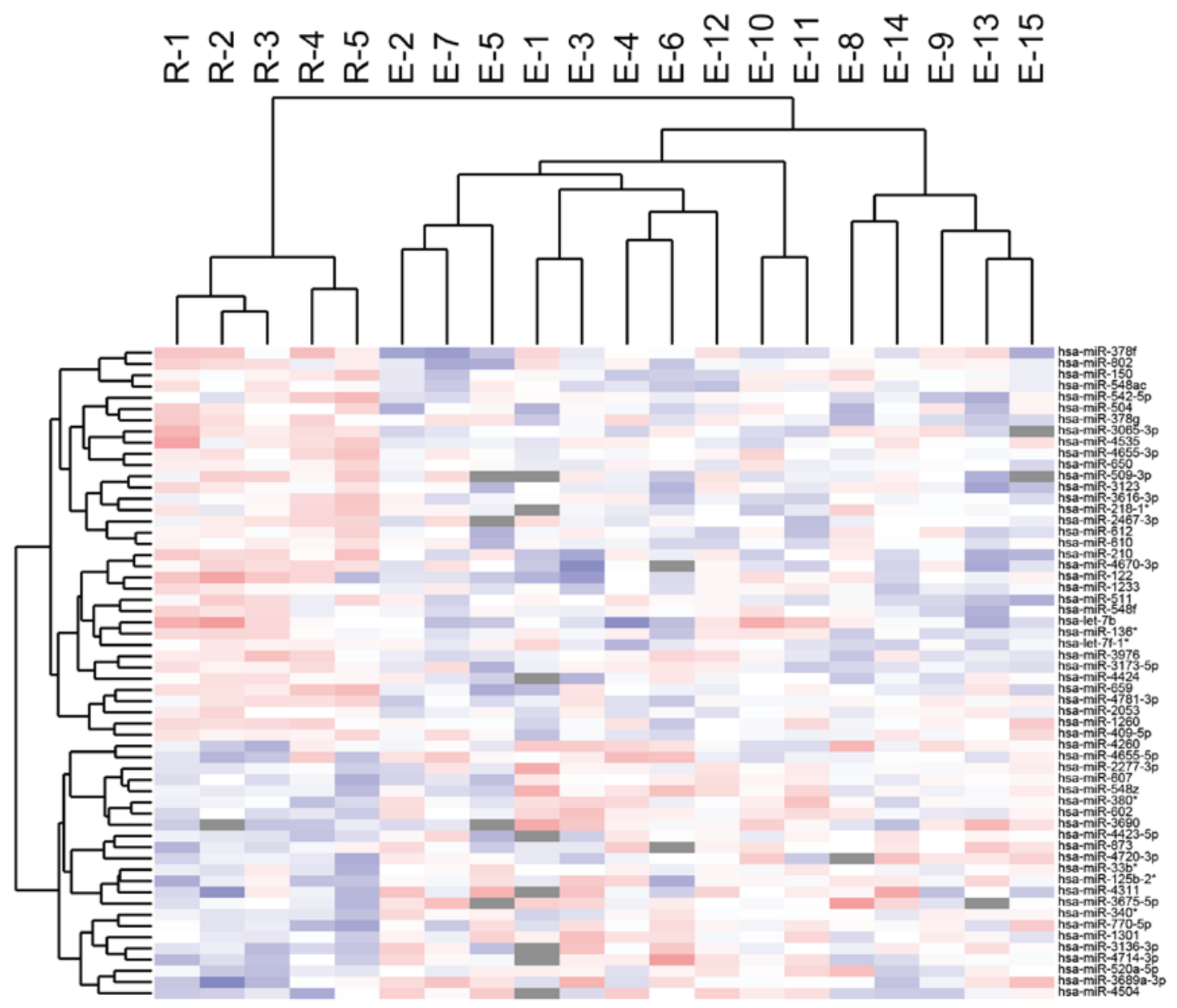

Figure 1. Hierarchical clustering of the treatment-effective and treatment-resistant groups. The patients with primary biliary cirrhosis were clustered according to their expression profiles of the 35 upregulated miRNAs and 23 downregulated miRNAs in the treatment-resistant group compared with the treatment-effective group. The miRNA clustering tree is presented on the left, with the sample-clustering tree at the top. The color scale presented illustrates the relative expression level of the miRNAs; red, high expression levels; blue, low expression levels. miRNA/miR, microRNA.

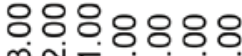 \\ miñ}
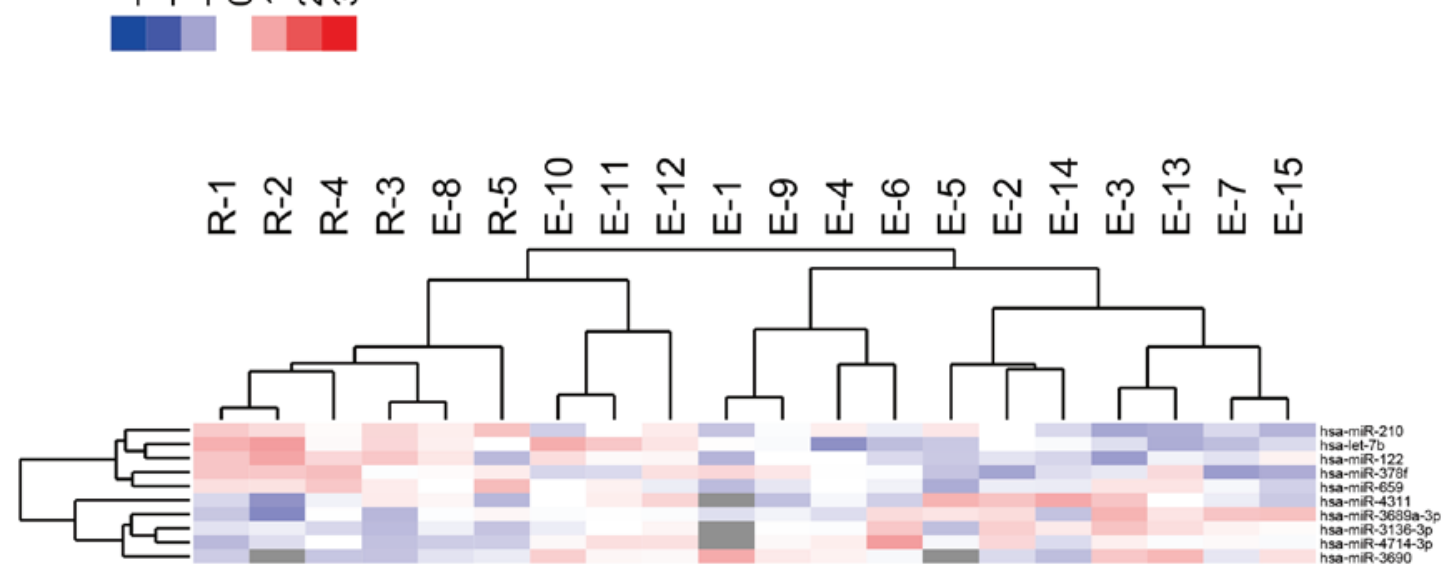

Figure 2. miRNAs that were markedly altered (greater than 1.5 -fold) were analyzed and clustered between the treatment-effective and treatment-resistant groups. The miRNA clustering tree is presented on the left, with the sample-clustering tree at the top. The color scale presented illustrates the relative expression level of the miRNAs; red, high expression levels; blue, low expression levels. miRNA/miR, microRNA. 
target and protein (33). Several miRNAs, including miR-125b, interact with the cytochrome P450, family 1, member A1 gene and induce drug resistance (33). In the current study, miR-125b was downregulated in the treatment-resistant group. The results of the current study are in agreement with a previous study that indicated that miRNAs are associated with drug resistance to UDCA or bezafibrate in patients with PBC (33).

In conclusion, identification of miRNA profiles is useful in characterizing the development of $\mathrm{PBC}$, and representative miRNAs, including miR-125b, let-7b and miR-520a-5p are suggested to be potential biomarkers for refractory PBC.

\section{References}

1. Morishita A and Masaki T: miRNA in hepatocellular carcinoma Hepatol Res 45: 128-141, 2015.

2. Suzuki H, Maruyama R, Yamamoto E and Kai M: Epigenetic alteration and microRNA dysregulation in cancer. Front Genet 4 258, 2013.

3. Bartel DP: MicroRNAs: Genomics, biogenesis, mechanism, and function. Cell 116: 281-297, 2004.

4. Krek A, Grün D, Poy MN, Wolf R, Rosenberg L, Epstein EJ, MacMenamin P, da Piedade I, Gunsalus KC, Stoffel M and Rajewsky N: Combinatorial microRNA target predictions. Nat Genet 37: 495-500, 2005.

5. Griffiths-Jones S: miRBase: MicroRNA sequences and annotation. Curr Protoc Bioinformatics Chapter 12, Unit 12.19: 1-10, 2010

6. Iborra M, Bernuzzi F, Invernizzi P and Danese S: MicroRNAs in autoimmunity and inflammatory bowel disease: Crucial regulators in immune response. Autoimmun Rev 11: 305-314, 2012.

7. Dai Y, Huang YS, Tang M, Lv TY, Hu CX, Tan YH, Xu ZM and Yin YB: Microarray analysis of microRNA expression in peripheral blood cells of systemic lupus erythematosus patients. Lupus 16: 939-946, 2007.

8. Pauley KM, Satoh M, Chan AL, Bubb MR, Reeves WH and Chan EK: Upregulated miR-146a expression in peripheral blood mononuclear cells from rheumatoid arthritis patients. Arthritis Res Ther 10: R101, 2008.

9. Neilson JR, Zheng GX, Burge CB and Sharp PA: Dynamic regulation of miRNA expression in ordered stages of cellular development. Genes Deve 21: 578-589, 2007.

10. Wu H, Neilson JR, Kumar P, Manocha M, Shankar P, Sharp PA and Manjunath N: MiRNA profiling of naive, effector and memory CD8 T cells. PloS One 2: e1020, 2007.

11. Zhou B, Wang S, Mayr C, Bartel DP and Lodish HF: miR-150, a microRNA expressed in mature B and T cells, blocks early B cell development when expressed prematurely. Proc Natl Acad Sci USA 104: 7080-7085, 2007.

12. Kaplan MM and Gershwin ME: Primary biliary cirrhosis. N Engl J Med 353: 1261-1273, 2005.

13. Shimoda S, Harada K, Niiro H, Shirabe K, Taketomi A, Maehara Y, Tsuneyama K, Nakanuma Y, Leung P, Ansari AA, et al: Interaction between Toll-like receptors and natural killer cells in the destruction of bile ducts in primary biliary cirrhosis. Hepatology 53: 1270-1281, 2011.

14. Hirschfield GM and Gershwin ME: The immunobiology and pathophysiology of primary biliary cirrhosis. Annu Rev Pathol 8: 303-330, 2013.

15. Corpechot $\mathrm{C}$, Chazouillères $\mathrm{O}$ and Poupon $\mathrm{R}$ : Early primary biliary cirrhosis: Biochemical response to treatment and prediction of long-term outcome. J Hepatol 55: 1361-1367, 2011.

16. Tanaka A, Hirohara J, Nakanuma Y, Tsubouchi $\mathrm{H}$ and Takikawa H: Biochemical responses to bezafibrate improve long-term outcome in asymptomatic patients with primary biliary cirrhosis refractory to UDCA. J Gastroenterol 50: 675-682, 2015
17. Padgett KA, Lan RY, Leung PC, Lleo A, Dawson K, Pfeiff J, Mao TK, Coppel RL, Ansari AA and Gershwin ME: Primary biliary cirrhosis is associated with altered hepatic microRNA expression. J Autoimmun 32: 246-253, 2009.

18. Chen X, Ba Y, Ma L, Cai X, Yin Y, Wang K, Guo J, Zhang Y, Chen J, Guo X, et al: Characterization of microRNAs in serum: A novel class of biomarkers for diagnosis of cancer and other diseases. Cell Res 18: 997-1006, 2008.

19. Poupon R: Primary biliary cirrhosis: A 2010 update. J Hepatol 52: 745-758, 2010.

20. Corpechot C, Abenavoli L, Rabahi N, Chrétien Y, Andréani T, Johanet $\mathrm{C}$, Chazouillères $\mathrm{O}$ and Poupon R: Biochemical response to ursodeoxycholic acid and long-term prognosis in primary biliary cirrhosis. Hepatology 48: 871-877, 2008.

21. Kuiper EM, Hansen BE, de Vries RA, den Ouden-Muller JW, van Ditzhuijsen TJ, Haagsma EB, Houben MH, Witteman BJ, van Erpecum KJ and van Buuren HR; Dutch PBC Study Group: Improved prognosis of patients with primary biliary cirrhosis that have a biochemical response to ursodeoxycholic acid Gastroenterology 136: 1281-1287, 2009.

22. Pares A, Caballería L and Rodes J: Excellent long-term survival in patients with primary biliary cirrhosis and biochemical response to ursodeoxycholic Acid. Gastroenterology 130: 715-720, 2006

23. Roderburg C, Benz F, Vargas Cardenas D, Koch A, Janssen J, Vucur M, Gautheron J, Schneider AT, Koppe C, Kreggenwinkel K, et al: Elevated miR-122 serum levels are an independent marker of liver injury in inflammatory diseases. Liver Int 35: 1172-1184, 2015.

24. Bihrer V, Friedrich-Rust M, Kronenberger B, Forestier N, Haupenthal J, Shi Y, Peveling-Oberhag J, Radeke HH, Sarrazin C, Herrmann E, et al: Serum miR-122 as a biomarker of necroinflammation in patients with chronic hepatitis $\mathrm{C}$ virus infection. Am J Gastroenterol 106: 1663-1669, 2011.

25. D'Alessandra Y, Devanna P, Limana F, Straino S, Di Carlo A, Brambilla PG, Rubino M, Carena MC, Spazzafumo L, De Simone M, et al: Circulating microRNAs are new and sensitive biomarkers of myocardial infarction. Eur Heart J 31: 2765-2773, 2010.

26. Zhang H, Su Y, Xu F, Kong J, Yu H and Qian B: Circulating microRNAs in relation to EGFR status and survival of lung adenocarcinoma in female non-smokers. PloS One 8: e81408, 2013.

27. Mayr C, Hemann MT and Bartel DP: Disrupting the pairing between let-7 and Hmga 2 enhances oncogenic transformation. Science 315: 1576-1579, 2007.

28. He J, Wu J, Xu N, Xie W, Li M, Li J, Jiang Y, Yang BB and Zhang Y: MiR-210 disturbs mitotic progression through regulating a group of mitosis-related genes. Nucleic Acids Res 41: 498-508, 2013 .

29. Han K, Zhao T, Chen X, Bian N, Yang T, Ma Q, Cai C, Fan Q, Zhou Y and Ma B: microRNA-194 suppresses osteosarcoma cell proliferation and metastasis in vitro and in vivo by targeting CDH2 and IGF1R. Int J Oncol 45: 1437-1449, 2014.

30. Swarbrick A, Woods SL, Shaw A, Balakrishnan A, Phua Y, Nguyen A, Chanthery Y, Lim L, Ashton LJ, Judson RL, et al: miR-380-5p represses p53 to control cellular survival and is associated with poor outcome in MYCN-amplified neuroblastoma. Nat Med 16: 1134-1140, 2010.

31. Le MT, Teh C, Shyh-Chang N, Xie H, Zhou B, Korzh V, Lodish HF and Lim B: MicroRNA-125b is a novel negative regulator of p53. Genes Dev 23: 862-876, 2009.

32. Yang L, Ma Z, Wang D, Zhao W, Chen L and Wang G: MicroRNA-602 regulating tumor suppressive gene RASSF1A is overexpressed in hepatitis B virus-infected liver and hepatocellular carcinoma. Cancer Biol Ther 9: 803-808, 2010.

33. Rukov JL, Wilentzik R, Jaffe I, Vinther J and Shomron N: Pharmaco-miR: Linking microRNAs and drug effects. Brief Bioinform 15: 648-659, 2014. 